\title{
Deep insights into the atmospheric Sodium hydroxide-hydrogen peroxide extraction process of hemicellulose in bagasse
}

\author{
Dong Chen ${ }^{1}$, Shibo Yang ${ }^{1}$, Lincai Peng ${ }^{2}$, Junhua Zhang ${ }^{1}$, Liang $\mathrm{He}^{2}$, Qingqing Guan ${ }^{2}$, \\ and Peng Zhang ${ }^{3}$
}

${ }^{1}$ Kunming University of Science and Technology

${ }^{2}$ Affiliation not available

3 Baiyun paper Co. Ltd., Zhumadian,

May 5, 2020

\begin{abstract}
The alkali dissolution characteristics and mechanism of hemicellulose in bagasse pith have been deeply analyzed. Firstly, the technical uncertainty of alkali-extraction and ethanol-separation process has been evaluated. The results showed that the effects of extraction process by-products (i.e., lignin and ash, etc) could be neglected and directly use ethanol precipitation without pre-neutralization for separation of hemicellulose in lye is reasonable. The kinetic behavior test showed that there exists an optimal alkali concentration $(\sim 100 \mathrm{~g} / \mathrm{L})$ to maximize the extraction yield of hemicellulose, attributing to the ratio of hydrodynamic diameter of hydrates and the intersheet distance of cellulosic microfibers. The activation energy of $22.19 \mathrm{KJ} / \mathrm{mol}$ indicated that the time-dependent dissolution process of hemicellulose could be mainly attributed to a diffusion controlled process. The developed model has been proved to be effective for prediction of hemicellulose concentration in the process-lye of atmospheric NaOH-H2O2 extraction of a bagasse pith under the experimental conditions studied.
\end{abstract}

\section{Graphical abstract}




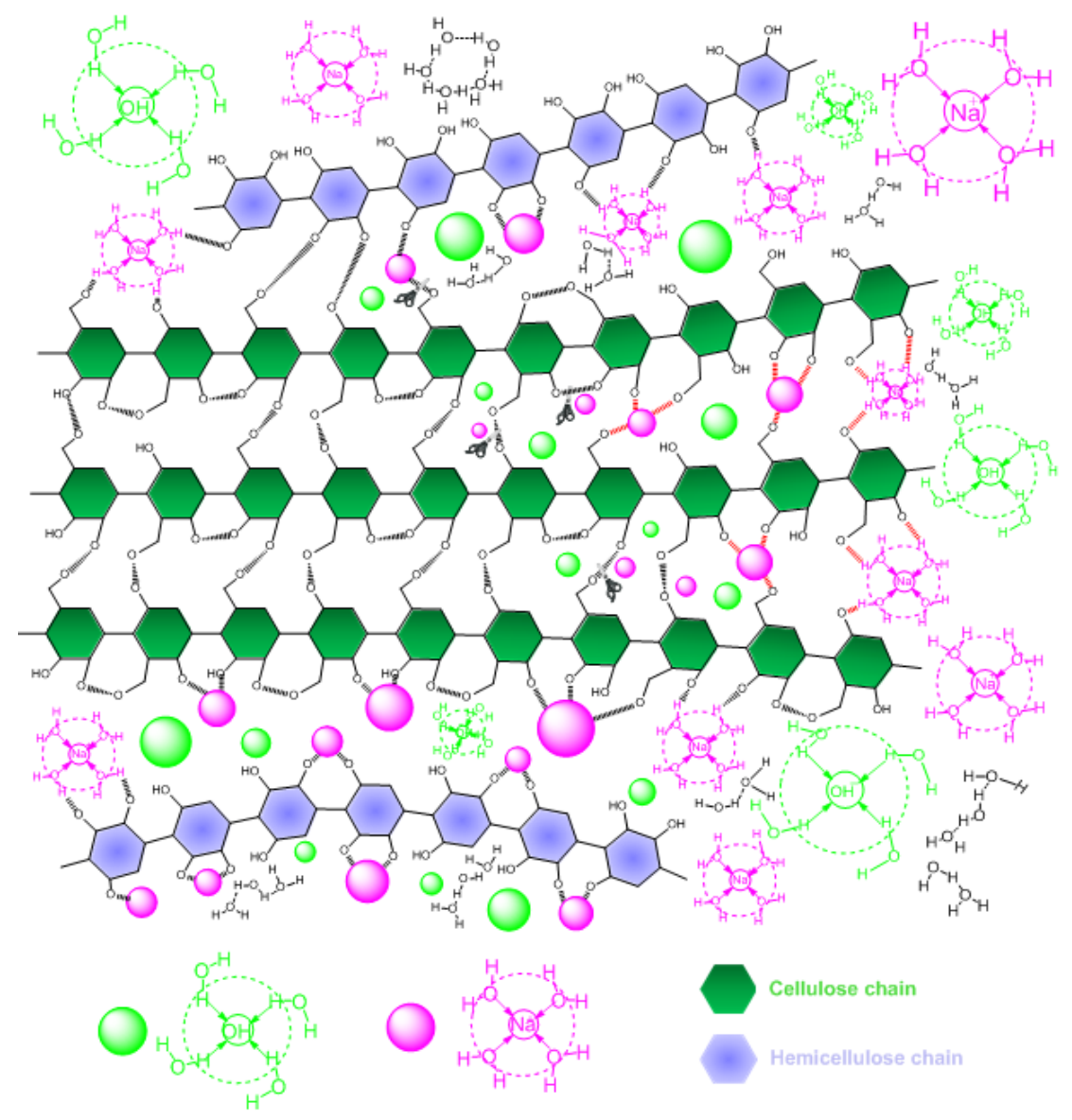

Schematic dissolution of hemicellulose from plant fibrous material during alkali treatment 

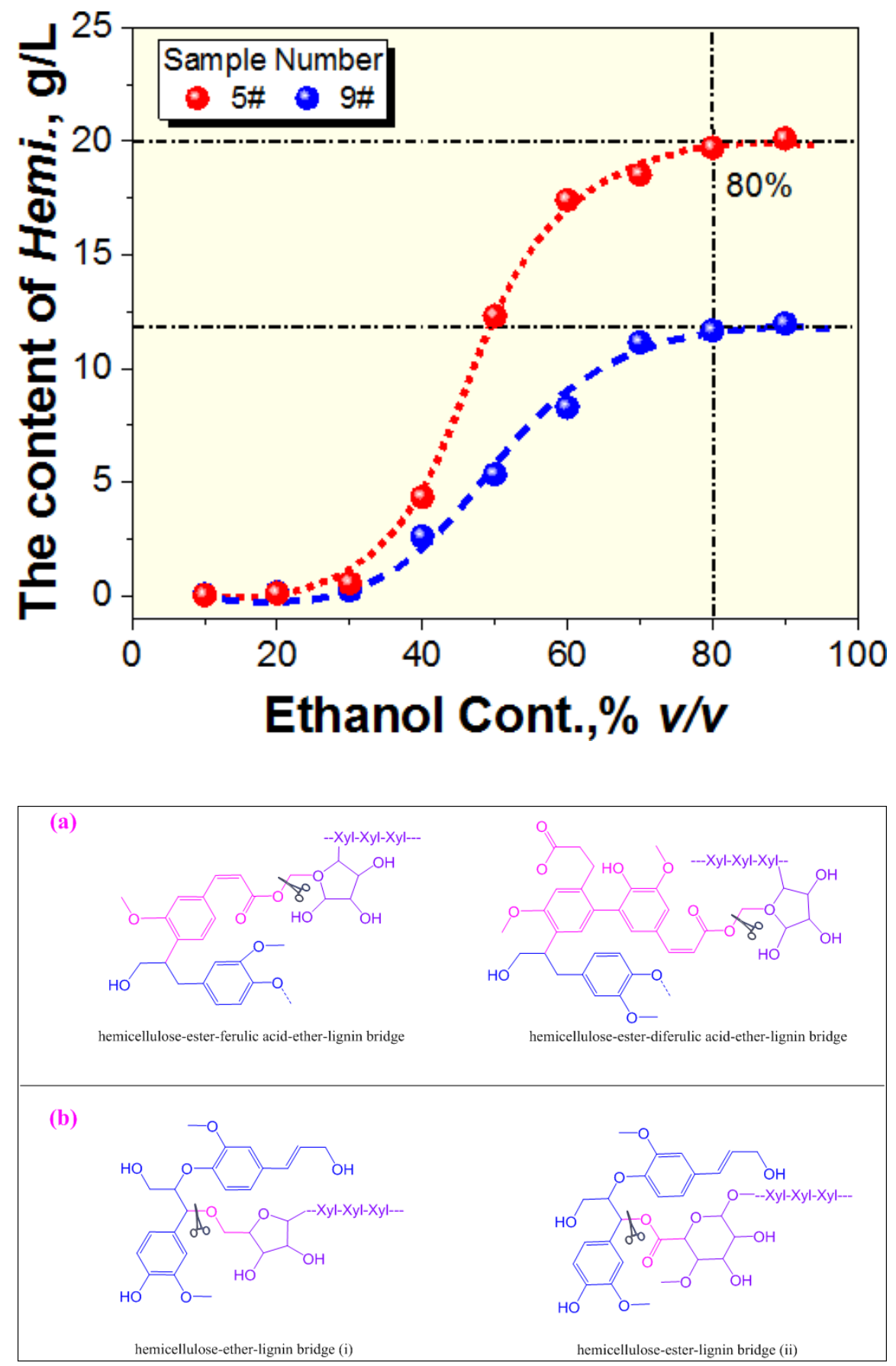
(A)

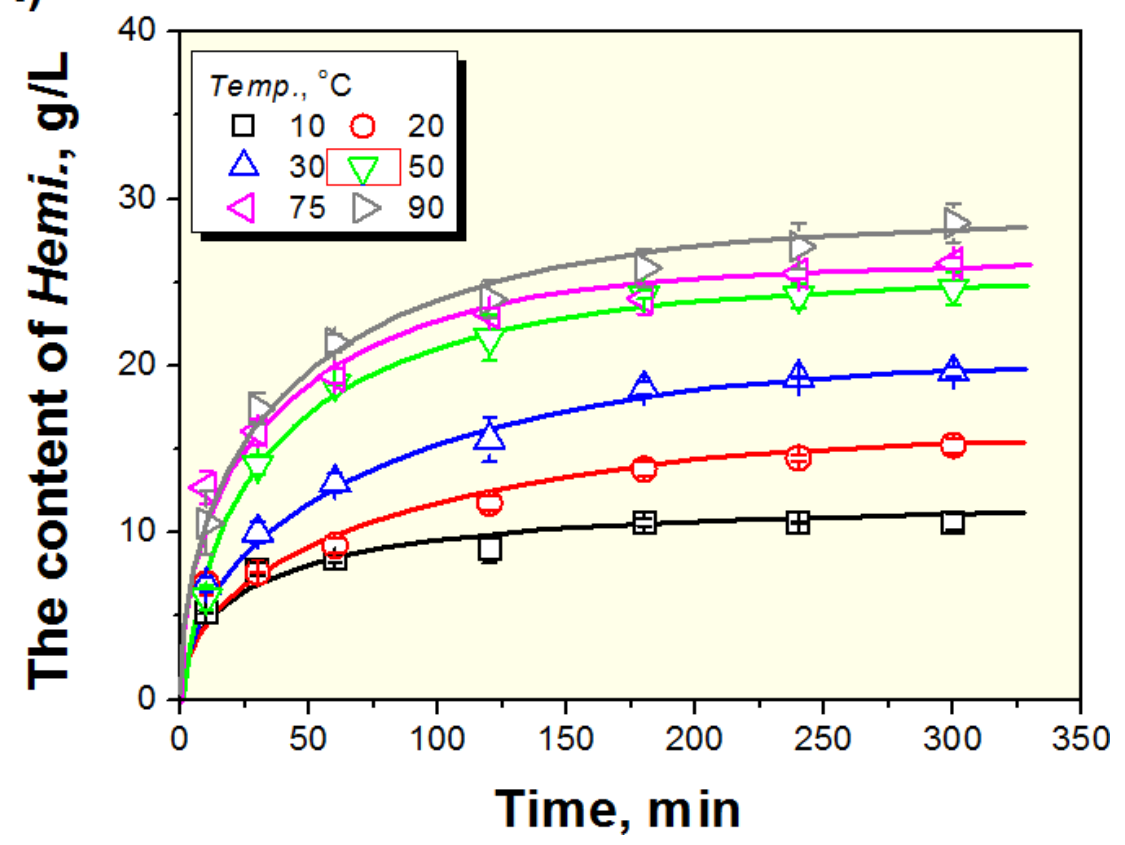

(B)

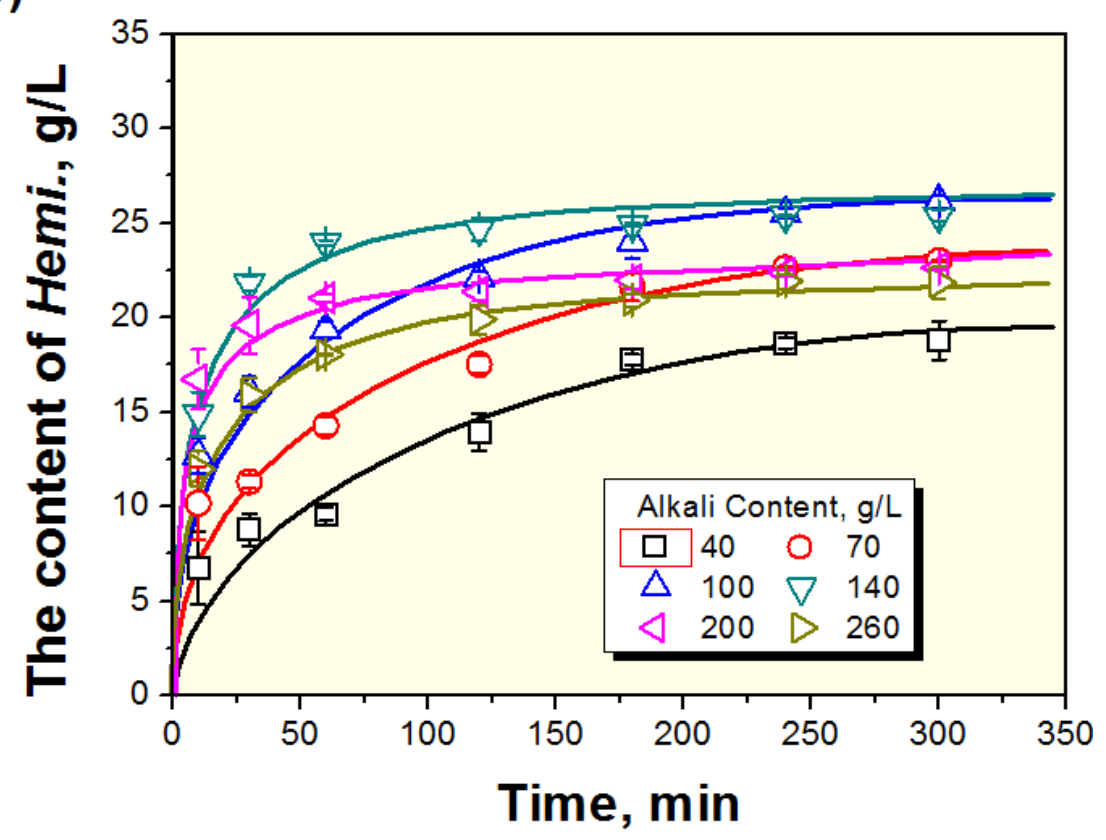


(C)

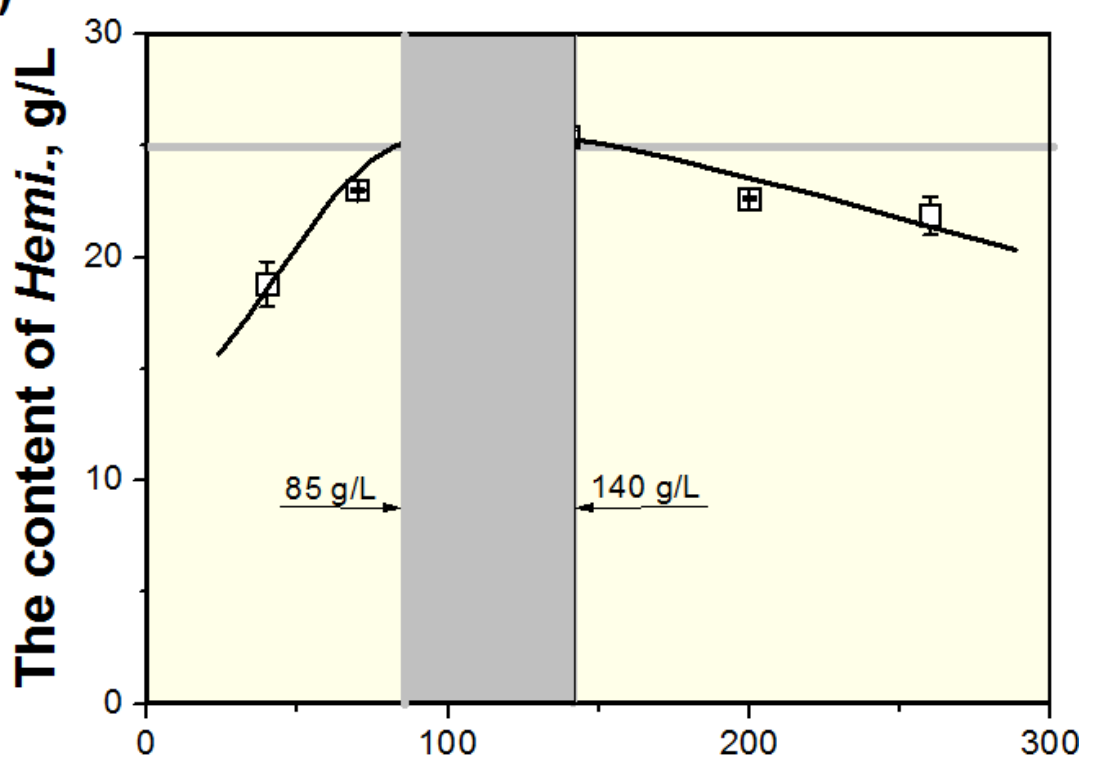

Alkali concentration, g/L

(D)

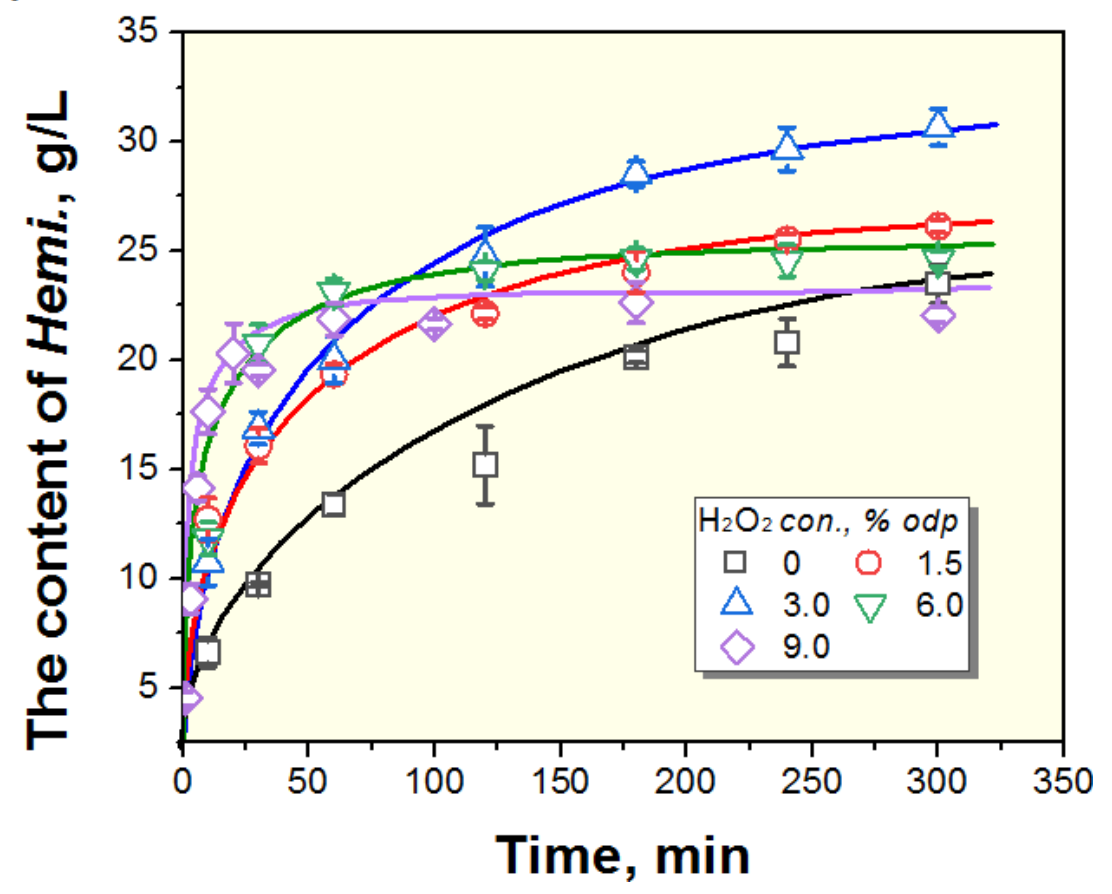




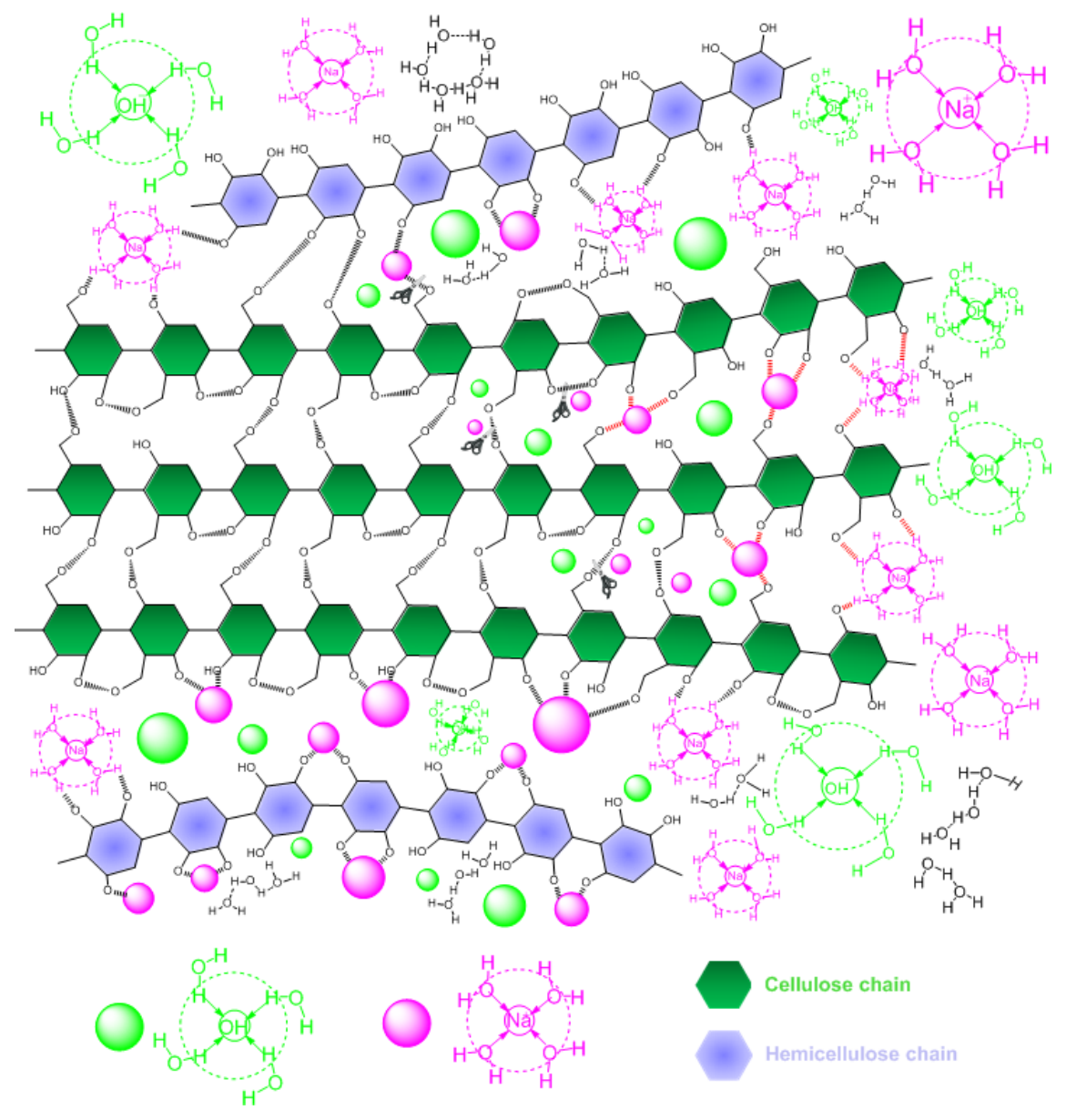



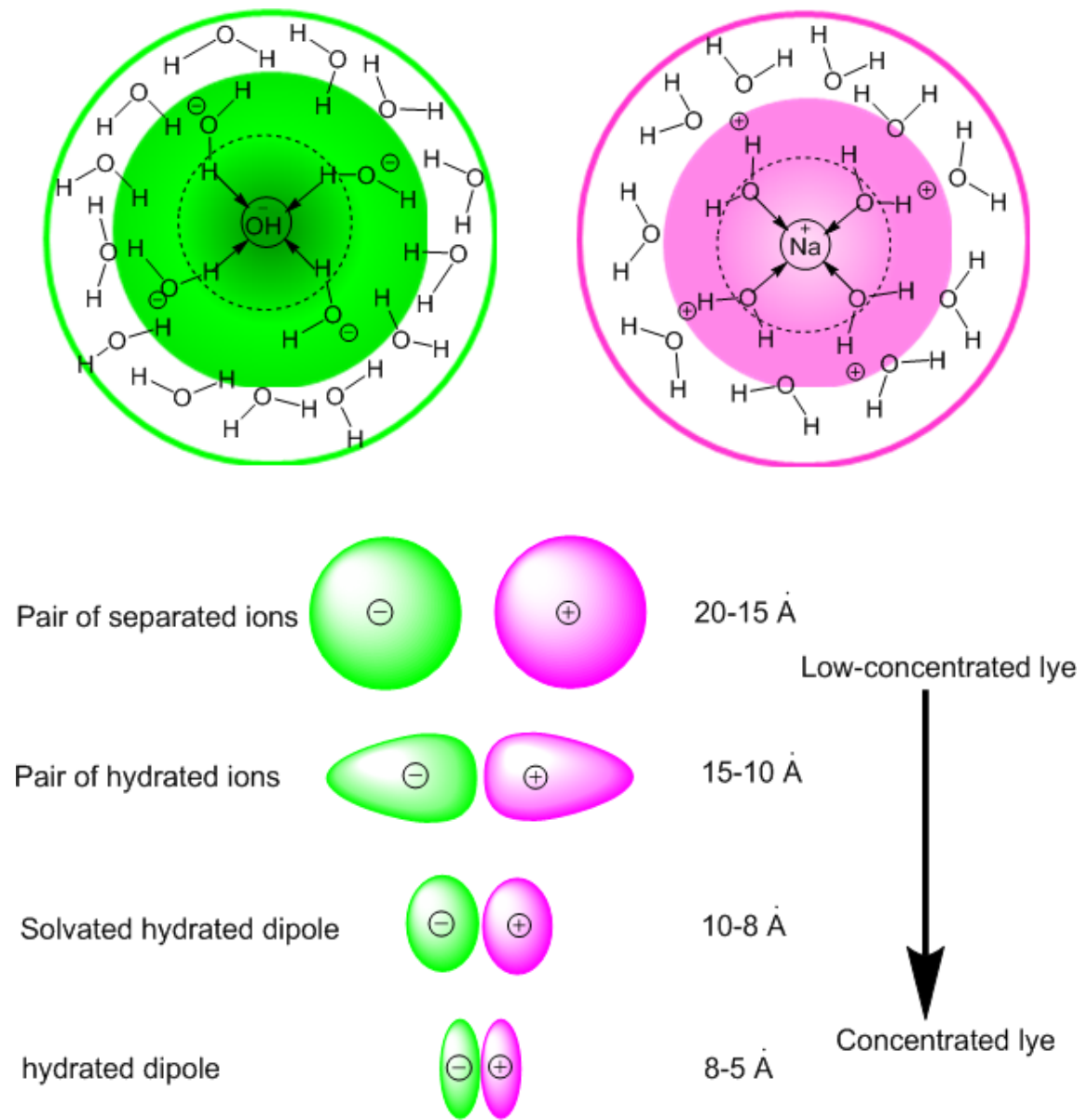
(A)

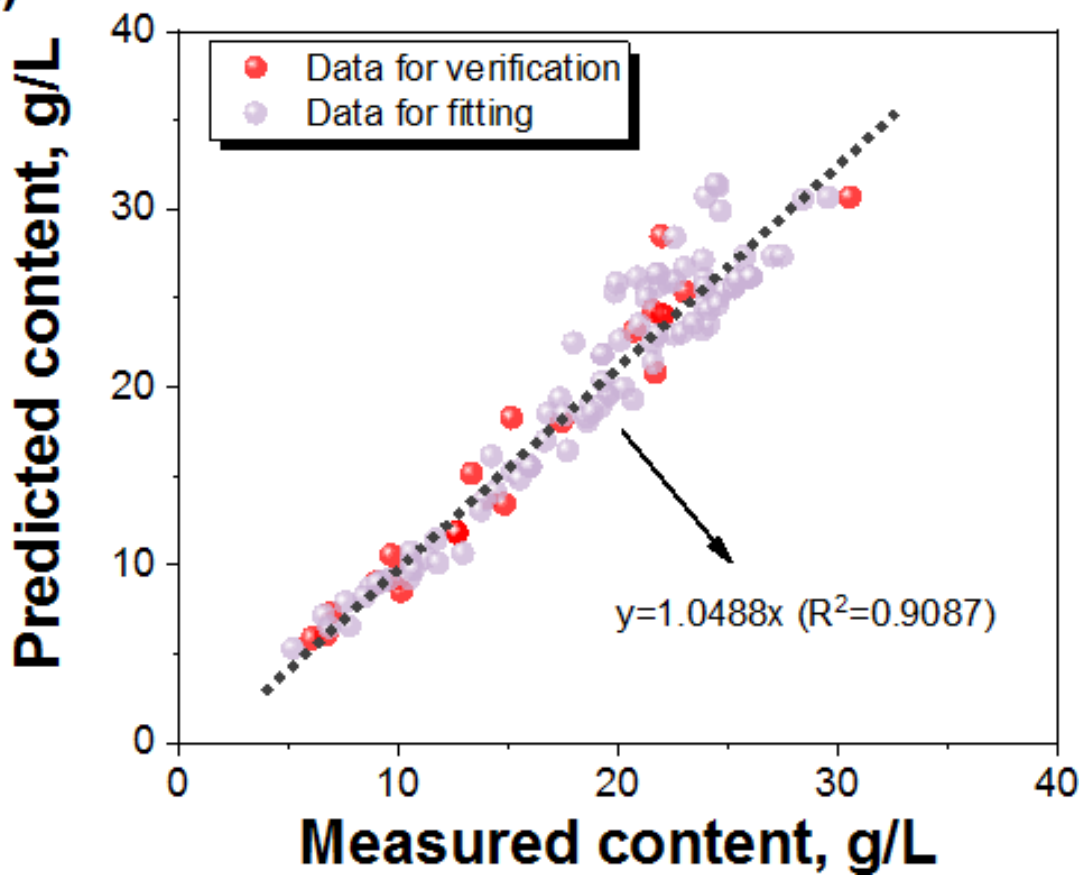

(B)

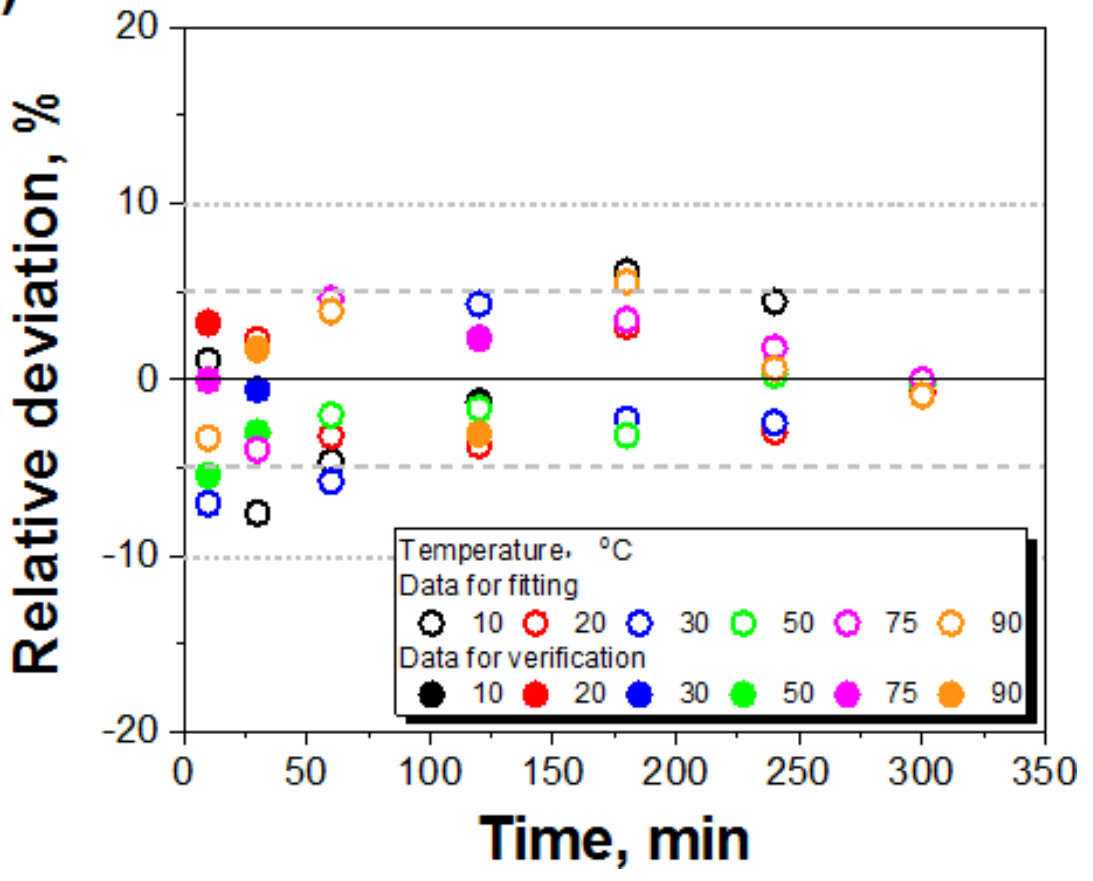


(C)

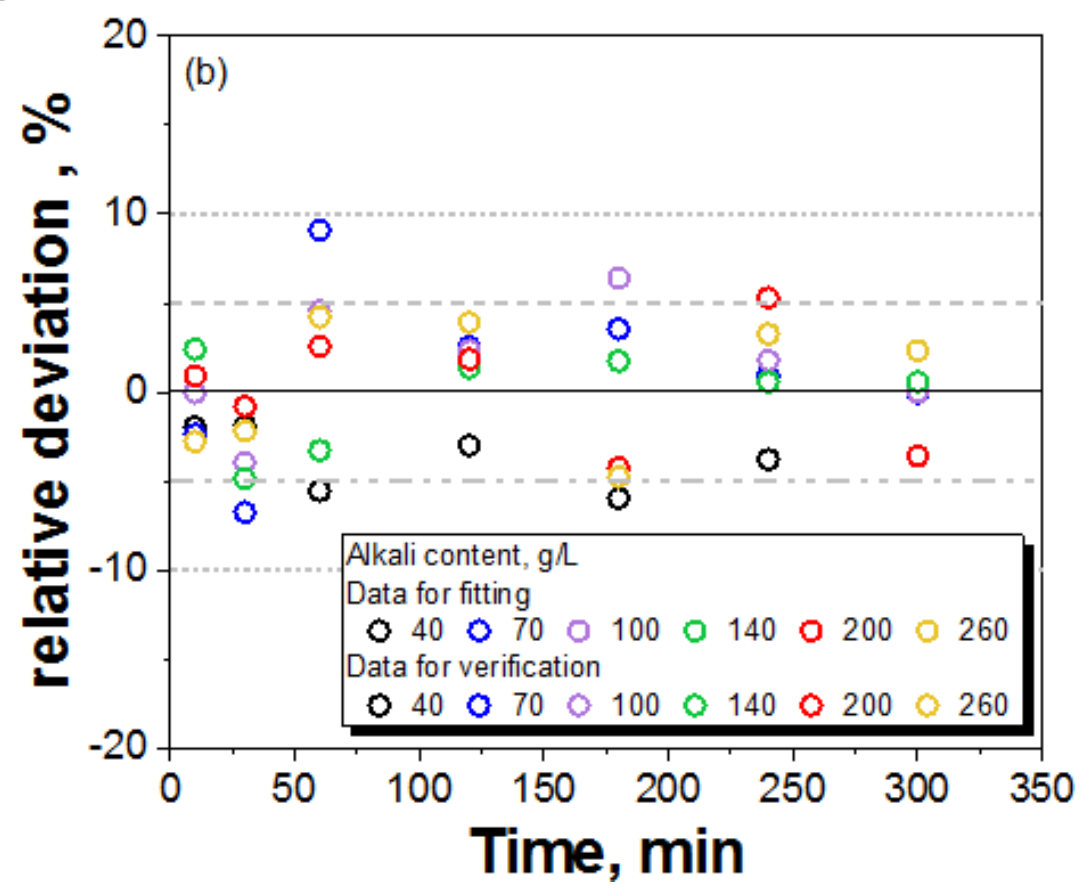

(D)

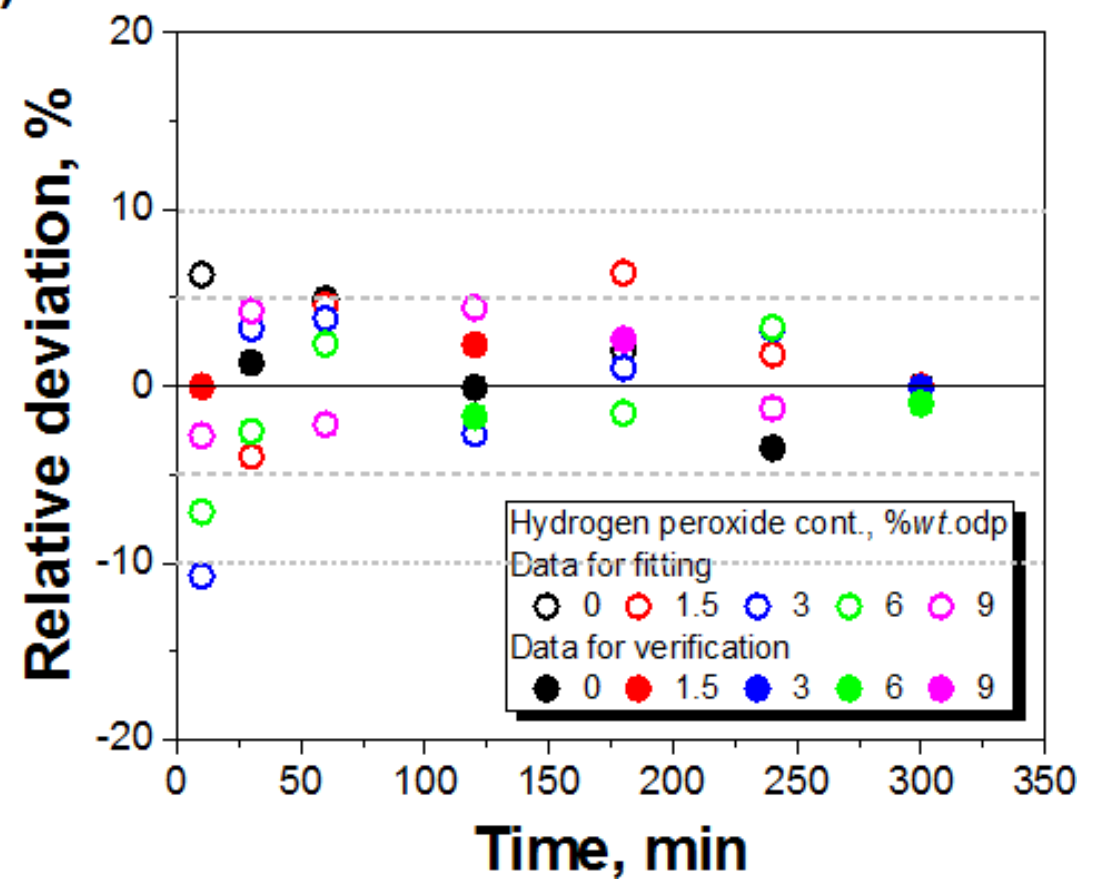

